\title{
Profound Impairment in Social Recognition and Reduction in Anxiety-Like Behavior in Vasopressin Vla Receptor Knockout Mice
}

\author{
Isadora F Bielsky*,', Shuang-Bao Hu', Kathleen L Szegda', Heiner Westphal ${ }^{\mathbf{3}}$ and Larry J Young' \\ 'Center for Behavioral Neuroscience, Department of Psychiatry, Emory University, Atlanta, GA, USA, ${ }^{2}$ Clinical Neuroendocrinology Branch, \\ National Institute of Mental Health, NIH, Bethesda, MD, USA, ${ }^{3}$ Laboratory of Mammalian Genes and Development, National Institute of Child \\ Health and Human Development, NIH, Bethesda, MD, USA
}

\begin{abstract}
Considerable evidence suggests that arginine vasopressin (AVP) is critically involved in the regulation of many social and nonsocial behaviors, including emotionality. The existence of two AVP receptors in the brain, namely the VIa and VIb subtypes, and the lack of clear pharmacological data using selective agonists or antagonists, make it difficult to determine which receptor is responsible for the AVP-mediated effects on behavior. Here we report the behavioral effects of a null mutation in the VIa receptor (VIaR) in male mice. Male mice lacking functional VIaR (VIaRKO) exhibit markedly reduced anxiety-like behavior and a profound impairment in social recognition. $\mathrm{V}$ IaRKO performed normally on spatial and nonsocial olfactory learning and memory tasks. Acute central administration of AVP robustly stimulated stereotypical scratching and autogrooming in wild-type (WT), but not $\mathrm{VIaRKO}$ males. AVP and oxytocin (OT) mRNA and OT receptor-binding levels were similar in WT and VIaRKO mice. Given the current findings, the VIaR may provide a novel potential pharmacological target for social and affective disorders including autism, and anxiety disorders.

Neuropsychopharmacology (2004) 29, 483-493, advance online publication, 26 November 2003; doi: I 0. I 038/sj.npp. I 300360
\end{abstract}

Keywords: vasopressin; VIa receptor; social recognition; anxiety; neuropeptide; knockout

\section{INTRODUCTION}

Arginine vasopressin (AVP), one of the first peptides to be characterized and extensively investigated, is both a neurohypophysial peptide released into the circulation from the posterior pituitary, and a neurotransmitter/neuromodulator distributed throughout the brain. Traditionally, research has focused on its peripheral actions, specifically the role of AVP in fluid homeostasis and blood pressure control. AVP also acts centrally within the CNS where it modulates a range of behaviors from learning and memory and responses to stressors to social behaviors. Beginning with the pioneering work of David de Wied in the late 1960s, early AVP behavioral studies were focused on its positive effects on avoidance learning and memory (reviewed in van Wimersma Greidanus et al, 1983; Engelmann et al, 1996). More recently, pharmacological studies have also demonstrated a role for AVP in the regulation of flank-marking behavior, paternal behavior,

\footnotetext{
*Correspondence: IF Bielsky, Yerkes National Primate Research Center, 954 Gatewood Drive, Atlanta, GA 30329, USA, Tel: + I 404727 8269, Fax: + I 404727 8070, E-mail: irapopo@emory.edu Received 16 July 2003; revised 23 October 2003; accepted 28 October 2003

Online publication: 29 October 2003 at http://www.acnp.org/citations/ Npp 102903033 | 6/default.pdf
}

social recognition, aggression, and affiliation, as well as anxiety-like behavior (Ferris et al, 1984, 1986; Le Moal et al, 1987; Winslow et al, 1993; Landgraf et al, 1995; Bamshad and Albers 1996; Liebsch et al, 1996; Everts and Koolhaas, 1997; Young et al, 1999; Parker and Lee, 2001; Pitkow et al, 2001).

While three receptor subtypes exist for AVP (V1a, V1b, and $\mathrm{V} 2$ ), the $\mathrm{V} 1 \mathrm{a}$ receptor (V1aR) has been thought to play the dominant role in regulating behavior. Until recently, the $\mathrm{V} 1 \mathrm{aR}$ was thought to be the only subtype expressed widely in the brain, while the V1b was localized primarily in the pituitary and the $\mathrm{V} 2$ in the kidney. V1aR antagonists produce marked effects on learning and memory and social behaviors. Intracerebroventricular (i.c.v.) and intraseptal administration of $\mathrm{V} 1 \mathrm{aR}$ antagonists block social recognition and AVP injections can rescue deficits in social recognition in Brattleboro rats that lack AVP (Dantzer et al, 1987; Engelmann and Landgraf, 1994; van Wimersma Greidanus and Maigret, 1996; Everts and Koolhaas, 1999). The effects of $\mathrm{V} 1 \mathrm{aR}$ antagonists on anxiety-related behaviors are contradictory, with different groups finding both anxiogenic and anxiolytic effects (Liebsch et al, 1996; Everts and Koolhaas, 1999). Septal administration of V1aR antisense oligos resulted in both impaired social recognition and a decrease in anxiety-related behavior in rats (Landgraf et al, 1995). 
The V1aR antagonists used in many of these studies are known to have low affinities for the V1bR and therefore it is conceivable that some of the behavioral effects produced by these compounds could be due to their effects on the V1bR. Recent in situ hybridization and immunohistochemical studies suggest that V1bR is not strictly localized to the pituitary, but may be widely distributed in the brain (Vaccari et al, 1998; Hernando et al, 2001). Thus, the relative contribution of the $\mathrm{VlaR}$ and VlbR on AVPmediated behaviors is less clear than once thought. The data supporting the role of $\mathrm{V} 1 \mathrm{bR}$ in social recognition and anxiety is sparse and inconsistent. Treatment with a novel, orally active V1b antagonist resulted in reduced anxietyrelated behavior while characterization of a V1b knockout mouse found no difference in anxiety-related behavior, but mildly impaired social recognition (Griebel et al, 2002; Wersinger et al, 2002).

The paradoxical and incongruous results of these earlier studies on the role of both V1bR and V1aR on cognition and anxiety necessitate further study of the mechanisms underlying the effects of AVP on these behaviors. Furthermore, AVP's role in avoidance learning, which is aversive in nature and the developing role of AVP in emotionality has lead to a vigorous debate into whether AVP effects on learning and memory are in fact a result of nonmnemonic changes in arousal state (Sahgal, 1984). We attempted to address these possibilities and controversies by testing the effects of a null mutation in the V1aR on emotionality, using anxiety tests, and two types of learning and memory. We used social recognition as a test for both social behavior, and nonaversive learning as well as the Morris water maze, which has not been clearly linked to AVP but is aversive in nature.

\section{MATERIALS AND METHODS}

\section{Animals}

Subjects were 2- to 5-month-old sexually naïve male mice bred in our laboratory. Briefly, the mice were produced by standard homologous recombination in which neo gene replacement deleted a $1.5 \mathrm{~kb}$ fragment of the V1aR gene starting at $31 \mathrm{bp}$ downstream of the translation start site and ending at $244 \mathrm{bp}$ downstream of exon I. Chimeric males were generated and mated to C57/BL6 females and germline transmission was achieved. These animals were backcrossed to $129 / \mathrm{SvJ}$ for five generations and experimental mice were produced from heterozygote crossings (Hu et al, 2003). The genotype of all animals was assessed using a PCR-based genotyping protocol. At 10 days of age, tails were clipped, the tissue was digested, and DNA was extracted and purified. PCR was performed on 1-2 $\mu \mathrm{g}$ of DNA using primer pairs $\mathrm{a} / \mathrm{b}$ and $\mathrm{c} / \mathrm{d}$. The knockout specific primer pair a/b produces a product of $430 \mathrm{bp}$ and the wildtype (WT) specific primer pair c/d produces a product of 369 bp. Primers used: a-AGAGGCTATTCGGCTATGACTG; bTTCGTCCAGATCATCCTGATC; c-GGGCGAGTTTCGTTCT GAGCATAC; d-GGTCATCTTCACTGTGCGGATCTTG. Animals were housed in groups of 3-5 for a majority of the work and then singly housed for the social recognition, olfactory habituation, and AVP administration studies. The animals were maintained in a $14 / 10 \mathrm{~h}$ light/dark cycle and were provided food and water ad libitum (Purina mouse chow; Purina mills, St. Louis, MO). Two groups of male mice were used in these studies: a WT group $N=8$, and a knockout null mutant $-I-(\mathrm{KO})$ group $N=9$. Heterozygotes were not included for purposes of controlling subject number. This allowed each test to be completed in 1 day and during the same part of the light/dark cycle, in an effort to reduce variability. All mice used in these studies were the products of heterozygous pairings in order to control for the possible confound of parental genotype. All experiments were performed in compliance with the rules and oversight of the Emory Institutional Animal Care and Use Committee.

\section{Behavioral Phenotyping}

All testing was performed in an isolated behavior room at $23-24^{\circ} \mathrm{C}$. Animals were brought to the testing room an hour before testing began, to acclimate to the environment. All behavior testing occurred in the first half of the light part of the light/dark cycle. All behaviors were videotaped and scored later by a single trained observer blind to genotype using a computer-assisted data acquisition system (Stopwatch + , http://www.cbn-atl.org/research/cores/behavioral software/stopwatch.cfm). For all anxiety tests the light in the room measured 300-400 lx. Each behavioral test was separated by at least $24 \mathrm{~h}$ and tests were performed in the order presented.

Elevated plus maze. The elevated plus maze apparatus consisted of two open arms and two closed arms each measuring $65 \times 6.25 \mathrm{~cm}$ and elevated $50 \mathrm{~cm}$ above the ground. The subject was placed in the center of the apparatus facing the open arm and its location was recorded for $5 \mathrm{~min}$. An arm entry was defined as the mouse having all four paws into the arm (Fernandes and File, 1996).

Open field. The open field apparatus consisted of a dark walled, circular open arena with a diameter measuring $67.5 \mathrm{~cm}$. The subject was placed in the arena, along the wall and its location was recorded for $5 \mathrm{~min}$. The critical measure was the time spent in the inner area of the arena ( $>5 \mathrm{~cm}$ away from any wall).

Light/dark box. The light/dark box apparatus consisted of a light, open topped, opaque, plexiglas box connected to a dark, closed topped, opaque, plexiglas box each measuring $27.5 \times 17.5 \times 12.5 \mathrm{~cm}$. The boxes were connected by a small opening that allows the subject to cross between them. The subject was placed in the light box and its location was recorded for $5 \mathrm{~min}$. The time spent in the light side of apparatus was considered the primary measure of the anxiety because of its sensitivity to anxiolytics (Hascoët et al, 2001). Light box entry was defined as the mouse having all four paws into the light box.

Morris water maze. The water maze apparatus consisted of a circular polypropylene pool that measured $95 \mathrm{~cm}$ in diameter. The pool was filled with water $\left(25^{\circ} \mathrm{C}\right)$ to a depth of $8 \mathrm{~cm}$. The water was made opaque by using water soluble, nontoxic white paint. A platform consisting of a white plastic column $(7.5 \mathrm{~cm}$ diameter $)$ was situated in the center 
of one quadrant of the pool and submerged $0.5 \mathrm{~cm}$ below water level. The pool was situated in a room with numerous and assorted extra-maze cues. The subject was acclimated for $2 \mathrm{~min}$ to the pool on the day before testing began. In four trials each day, subjects were placed at all of four systematically varied positions along the edge of the pool. The intertrial interval (ITI) was $10 \mathrm{~min}$. We measured latency to locate the submerged platform. If the subject did not find the platform in $60 \mathrm{~s}$, it was placed on the platform and allowed to remain there for $15 \mathrm{~s}$. Sessions were repeated on 5 successive days during the first half of the light part of the light/dark cycle. On the sixth day the subjects were exposed to a probe trial of $60 \mathrm{~s}$ in which the platform was removed and the time spent swimming in each of the quadrants was recorded (adapted from Morris, 1984).

Forced swim. The forced swim apparatus was a clear glass cylinder $13.75 \mathrm{~cm}$ in diameter and $18.13 \mathrm{~cm}$ high that was filled to a depth of $11.9 \mathrm{~cm}$ with water $\left(25^{\circ} \mathrm{C}\right)$. The subject was placed in the water for $6 \mathrm{~min}$. The time spent floating $v s$ swimming during the last 3 min was recorded (Porsolt $e t$ al, 1978; Sanchez and Meier, 1997).

Baseline acoustic startle and prepulse inhibition (PPI). Startle and sensorimotor gating was assessed using acoustic startle and PPI using a modified version of that used by Dulawa and Geyer (2000). For acoustic startle, mice were exposed to pulses of $90,100,110$, and $120 \mathrm{~dB}$ in a pseudorandomized order with 20 presentations of each stimulus and an ITI of $30 \mathrm{~s}$. For PPI, mice received multiple $40 \mathrm{~ms} 120 \mathrm{~dB}$ tones (startle alone) intermittently proceeded by four different prepulse signals (from 6 to $14 \mathrm{~dB}$ above background). Trials were presented in pseudorandom order with an ITI of $30 \mathrm{~s}$ and an interstimulus interval (between prepulse and pulse) of $100 \mathrm{~ms}$. For both acoustic startle and PPI the background noise was $60 \mathrm{~dB}$ and the maximum startle was considered to be the peak startle within $200 \mathrm{~ms}$ of the stimulus.

Social recognition. Animals were individually housed for 10 days before testing to permit the establishment of a homecage territory. The procedure was the same as previously used in Ferguson et al (2000). Briefly, the subject was exposed to the same ovariectomized adult female mouse for $1 \mathrm{~min}$ over four trials with an ITI of $10 \mathrm{~min}$. During the fifth dishabituation trial, the subject was exposed to a novel ovariectomized female for $1 \mathrm{~min}$. The time spent in olfactory investigation for each trial was recorded. Olfactory investigation was defined as direct, active, olfactory exploration of the stimulus female; specifically nosing and sniffing of the head and anogenital regions, closely following, and pursuit. Each female was used only once each day. Mice displaying normal social recognition show a decline in investigation upon subsequent exposure to the same individual; and in the dishabituation trial, investigation times are similar to the initial exposure to the first individual.

Olfactory habituation. Animals were individually housed for at least 10 days before testing and olfactory habituation was performed 7 days after social recognition. The procedure was used previously by Ferguson et al (2000). Briefly, a small plastic cartridge was packed with cotton scented with $10 \mu \mathrm{l}$ of anise extract. This was placed in the home cage of the subject for 1 min over four trials, with an ITI of $10 \mathrm{~min}$. In the fifth dishabituation trial the subject was presented with a cotton ball scented with $10 \mu \mathrm{l}$ of coconut extract. The time spent in olfactory investigation for each trial was recorded. Olfactory investigation was defined as direct nasal contact with the cartridge.

Data and statistical analysis. For the plus maze, open field, and light/dark box, the time spent in the open arm, open field, and light box was compared between genotypes using a Student's $t$-test. The number of closed arm entries in the plus maze and the number of nose pokes in the light box were compared between genotype using a Student's $t$-test. For the forced swim task, the amount of time spent floating was compared between genotype using a Student's $t$-test. For baseline startle and PPI, two-way ANOVAs, with genotype and pulse/prepulse intensity as factors, with repeated measures on pulse/prepulse intensity were performed. For the acquisition portion of the Morris water maze, social recognition, and olfactory habituation test, two-way ANOVAs, with genotype and day/trial as factors, with repeated measures on day/trial were performed. For the retention, probe trial, portion of the Morris water maze, a two-way ANOVA, with genotype and quadrant as factors, with repeated measures on quadrant was performed. If a significant main or interaction effect was detected, a Neuman-Keuls post hoc analysis was performed $(\alpha=0.05)$.

\section{Pharmacological Studies}

After completion of the behavioral testing animals were implanted with an intraventricular cannula as described previously in Ferguson et al (2000). Briefly, animals were anaesthetized with ketamine and domitor, placed in the stereotaxic apparatus and implanted intraventricularly with a 26-gauge stainless-steel infusion cannula (Plastics One, Roanoke, VA). Injections began 3-5 days after surgery and were made using a $10 \mu \mathrm{l}$ Hamilton syringe connected to a 33-gauge injection needle via PE tubing. Either artificial cerebrospinal fluid (CSF) or $10 \mathrm{ng}$ doses of AVP dissolved in CSF were delivered to awake, restrained subjects in a $4 \mu \mathrm{l}$ volume infused over $60 \mathrm{~s}$. Subjects were recorded for $10 \mathrm{~min}$ immediately after the injection cannula was removed and behavior was analyzed. All animals received infusions of both AVP and control vehicle with at least 2 days in between treatments in a counter-balanced design. Cannula placement was confirmed with post-mortem examination of cryostat sections, and only those animals with correct placement were included for analysis.

Data and statistical analysis. Time spent scratching and autogrooming was analyzed using a two-way ANOVA, with genotype and treatment as factors, with repeated measures on the treatment factor. If a significant interaction or main effect was detected, a Neuman-Keuls post hoc analysis was performed $(\alpha=0.05)$. 


\section{Receptor Autoradiography and In situ Hybridization}

For the radioligand receptor binding and in situ hybridization, brains from all experimental subjects were removed, flash frozen, sectioned at $20 \mu \mathrm{m}$, and slide mounted into four serial sets for each experimental animal. One set each was used for V1aR binding, oxytocin receptor (OTR) binding, OT expression, and AVP expression. Sections were processed for receptor autoradiography using $\mathrm{I}^{125}$-labeled $\mathrm{d}\left(\mathrm{CH}_{2}\right)_{5}\left[\mathrm{Tyr}(\mathrm{Me})_{2},-\mathrm{Tyr}-\mathrm{NH}_{2}^{9}\right]$ ornithine vasotocin (NEN Life Sciences Products, Boston, MA) for the OTR and (Phenylacetyl ${ }^{1}, 0-M e-D-$ Tyr $^{2},\left[{ }^{125}{\left.\left.\mathrm{I}-\mathrm{Arg}^{6}\right]-\right)}\right.$ ) vasopressin (linear), V$1 \mathrm{~A}$ antagonist (NEN Life Science Products, Boston, MA) for the VlaR as described previously (Nishimori et al, 1996; Young et al, 1997). Slides were exposed to BioMax MR film (Kodak) for $48 \mathrm{~h}$. Results from the V1aR autoradiography were used to confirm genotype. Sections were processed for AVP and OT expression using ${ }^{35} \mathrm{~S}$-labeled antisense oligos as described previously (Nishimori et al, 1996; Young et al, 1996). Slides were exposed to Bio Max MR film (Kodak) for $1-2 \mathrm{~h}$.

Data and statistical analysis. Film autoradiograms and in situ hybridization images were analyzed using the AIS program (Imaging Research Inc., St Catharines, ON, Canada). For the receptor-binding autoradiograms, optical densities were converted to $\mathrm{dpm} / \mathrm{mg}$ of tissue equivalents using ${ }^{125} \mathrm{I}$ microscales (Amersham, Arlington Heights, IL). Specific binding in the lateral septum and medial amygdala was obtained by subtracting the nonspecific binding from the total binding for each section and area. In situ hybridization was quantified using optical density and specific hybridization was obtained by subtracting the optical density of the area adjacent to the region being measured from that of the paraventricular nucleus (PVN) for each section. All sections were analyzed by a trained experimenter blind to genotype and each region of interest was measured bilaterally from at least two sections. Values from WT and KO subjects were compared using Student's $t$-test $(\alpha=0.05)$.

\section{RESULTS}

\section{Behavior Testing}

Elevated plus maze, open field, light/dark box, and forced swim. Male V1aRKO mice demonstrated significantly less anxiety-like behaviors in all tests for anxiety behavior. V1aRKO males spent more time in the open arms of the elevated plus maze than did their WT littermates $(p<0.05)$ (Figure 1a). There were no significant genotype differences in overall activity in the elevated plus maze as measured by the number of closed arm entries (Figure 1b). Male V1aRKO mice spent significantly more time in the inner area of the open field arena $(p<0.05)$ (Figure 1e) and significantly more time in the light box of the light/dark box $(p<0.05)$ (Figure 1c) than did their WT littermates. There were no genotype differences in exploratory behavior in the light/ dark box task as measured by the number of nose pokes into the light box (Figure 1d). Differences in anxiety-like behaviors in the V1aRKO were not associated with differences in depressive-like behavior as there was no difference between genotypes in the forced swim test (Figure 1f).

Morris water maze, baseline acoustic startle, and PPI. There were no differences between the V1aRKO and WT subjects in the Morris water maze, baseline acoustic startle, and PPI. For the learning portion of the Morris water maze, a significant main effect of test day $\left(\mathrm{F}_{4,15}=6.132, p<0.001\right)$ but not genotype $\left(F_{1,15}=0.338\right.$, NS) was detected for the latency to find the submerged platform. No significant interaction was detected $\left(\mathrm{F}_{4,15}=0.315\right.$, NS). Post hoc analysis revealed that both the $\mathrm{V} 1 \mathrm{aRKO}$ and $\mathrm{WT}$ mice showed a significantly reduced latency to find the platform on test days 4 and 5 (both $p<0.05$ ) as compared to test day 1 (Figure 2a). For the probe trial of the Morris water maze, a significant main effect of quadrant $\left(\mathrm{F}_{3,15}=16.998, p<0.001\right)$ but not genotype $\left(\mathrm{F}_{1,15}=0.560\right.$, NS) was detected for the time spent in each quadrant. No significant interaction effect was detected $\left(\mathrm{F}_{3,15}=2.275\right.$, NS). Post hoc analysis revealed that both $\mathrm{V} 1 \mathrm{aRKO}$ and $\mathrm{WT}$ mice spent significantly more time in the target quadrant as compared to all other quadrants (both $p<0.001$ ) (Figure 2b). Both the V1aRKO and WT subjects showed similar baseline acoustic startle and PPI, as there were no main effect of genotype in either test $\left(F_{1,15}=0.595, \mathrm{NS} ; \mathrm{F}_{1,15}=0.975, \mathrm{NS}\right)$. There was a significant main effect of pulse or prepulse intensity in both test $\left(\mathrm{F}_{3,15}=69.656, p<0.001 ; \mathrm{F}_{3,15}=49.737, p<0.001\right)$ as both genotypes showed significantly increased startle with increasing pulse and prepulse intensity. There was no interaction effect in either test $\left(\mathrm{F}_{3,15}=0.414, \quad \mathrm{NS}\right.$; $\mathrm{F}_{3,15}=0.707, \mathrm{NS}$ ) (Figure $3 \mathrm{a}$ and $\mathrm{b}$ ).

Social recognition and olfactory habituation. V1aRKO mice showed a significant deficit in social recognition. Significant main effects for trial $\left(\mathrm{F}_{4,15}=17.827, p<0.001\right)$ and genotype $\left(F_{1,15}=6.450, p<0.05\right)$ were detected for time spent in olfactory investigation of a stimulus female. A significant interaction effect was also detected $\left(F_{4,15}=9.637\right.$, $p<0.001)$. Post hoc analysis revealed that WT mice showed a significant decrease in social olfactory investigation upon subsequent presentations of the same female in trials 3 $(p<0.01)$ and $4(p<0.001)$ as compared to trial 1 . V1aRKO subjects had significantly impaired social recognition and never demonstrated a significant reduction in olfactory investigation in any of the trials (Figure 4a). The olfactory investigation decrease seen in the WT subjects in trials 3 and 4 was not due to a general decrease in olfactory investigation because presentation of a novel female during trial 5 resulted in a similar amount of investigation as trial 1 with the original female.

The lack of social recognition in the V1aRKO was not due to a general deficit in olfaction as both V1aRKO and WT subjects habituated to repeated presentations of a same scented cotton ball. A significant effect of trial $\left(\mathrm{F}_{4,15}=93.201, p<0.001\right)$ but not genotype $\left(\mathrm{F}_{1,15}=1.274\right.$, NS) was detected for the time spent in olfactory investigation. No interaction effect was detected $\left(\mathrm{F}_{4,15}=1.130, \mathrm{NS}\right)$. Post hoc analysis revealed that both $\mathrm{V} 1 \mathrm{aRKO}$ and WT mice showed a significant decrease in time spent in olfactory investigation of a same scented cotton ball on trials 2,3 , and $4(p<0.001)$ (Figure 4b). Again, this was not due to a 
a
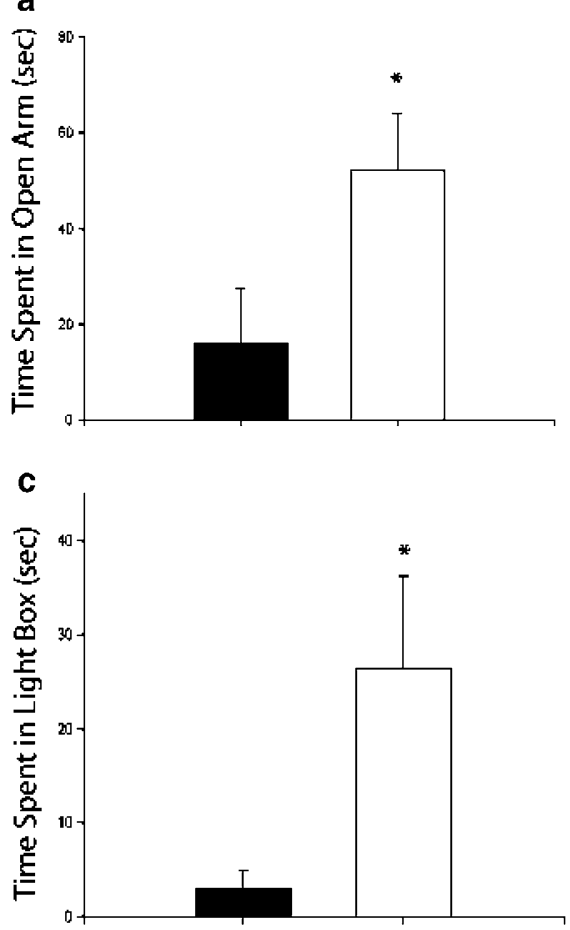

e

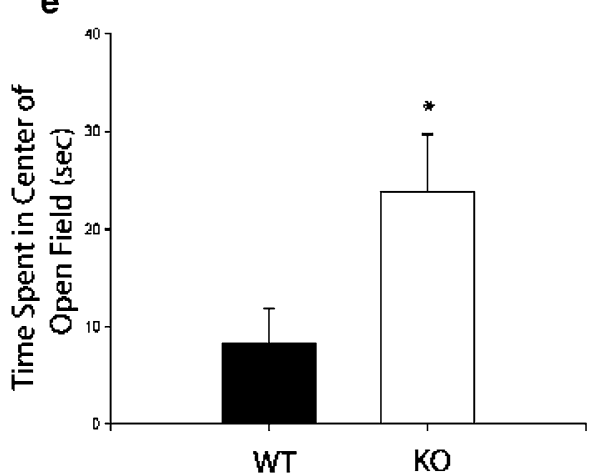

b

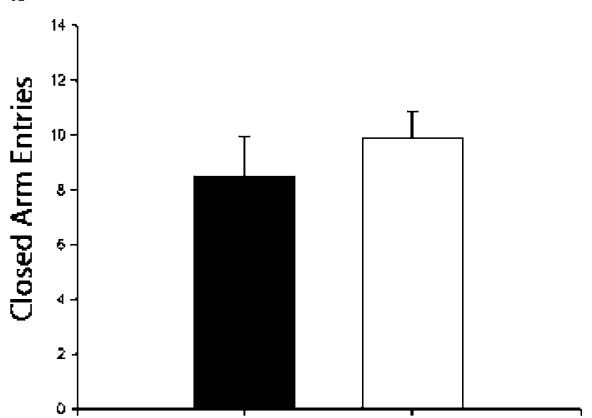

d

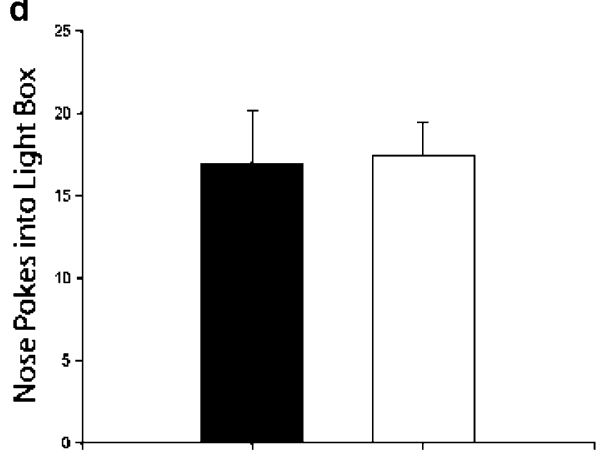

f

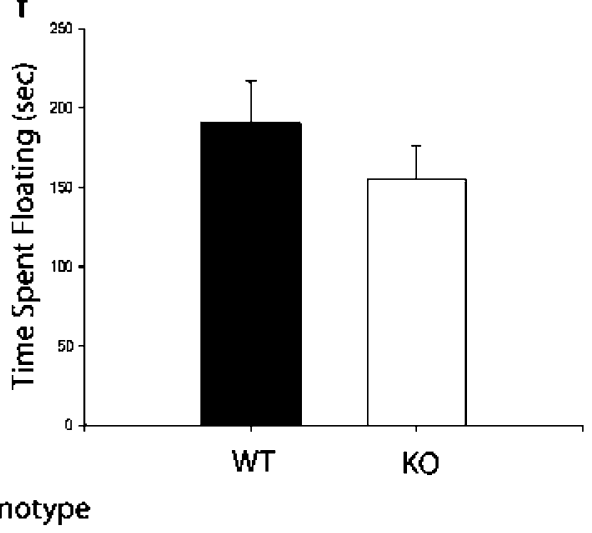

Figure I Anxiety-related behaviors for WT (filled bars, $N=8$ ) and VI aRKO (open bars, $N=9$ ) male mice. $V$ I aRKO animals spent significantly more time in the open arm of the elevated plus maze (a), in the light box of the light/dark box test (c), and in the center area of the open field arena (e) compared to their WT littermates. There was no difference between the VIaRKO and WT mice in the number of closed arm entries in the elevated plus maze (b), in the number of nose pokes in the light/dark box test (d), or in the time spent floating $(f)$ in the forced swim test. Error bars represent SEM. * $p<0.05$.

general decrease in olfactory investigation because presentation of a novel scented cotton ball on trial 5 resulted in a similar amount of investigation as trial 1 with the original scent. There was no genotypic difference in the amount of initial investigation in either test.

\section{Pharmacological Studies}

Central AVP administration in WT subjects resulted in a large increase in scratching and autogrooming behaviors as compared to vehicle injection (Figure 5a and b). Significant main effects of genotype and treatment for scratching $\left(\mathrm{F}_{1,15}=43.346, p<0.001\right.$ and $\left.\mathrm{F}_{1,15}=44.190, p<0.001\right)$ and autogrooming $\left(\mathrm{F}_{1,15}=50.415, p<0.001\right.$ and $\mathrm{F}_{1,15}=82.550$, $p<0.001)$ were detected as well as interaction effects for both measures $\left(\mathrm{F}_{1,15}=44.826, p<0.001\right.$ and $\mathrm{F}_{1,15}=55.129$, $p<0.001$ respectively). Post hoc analysis revealed that AVP treatment resulted in a significant increase in scratching and autogrooming in WT mice only $(p<0.001)$. There was no difference in scratching and autogrooming between AVP and vehicle treatment in the V1aRKO subjects and vehicle treatment in the WT subjects.

\section{Receptor Autoradiography and In Situ Studies}

There were no apparent effects of the null mutation in the V1aR on OT $(p>0.05)$ and AVP $(p>0.05)$ mRNA expression in the PVN and no effects on the binding or distribution of brain OTR. Average optical density for OT and AVP mRNA in the WT subjects was $0.15 \pm 0.03$ and 

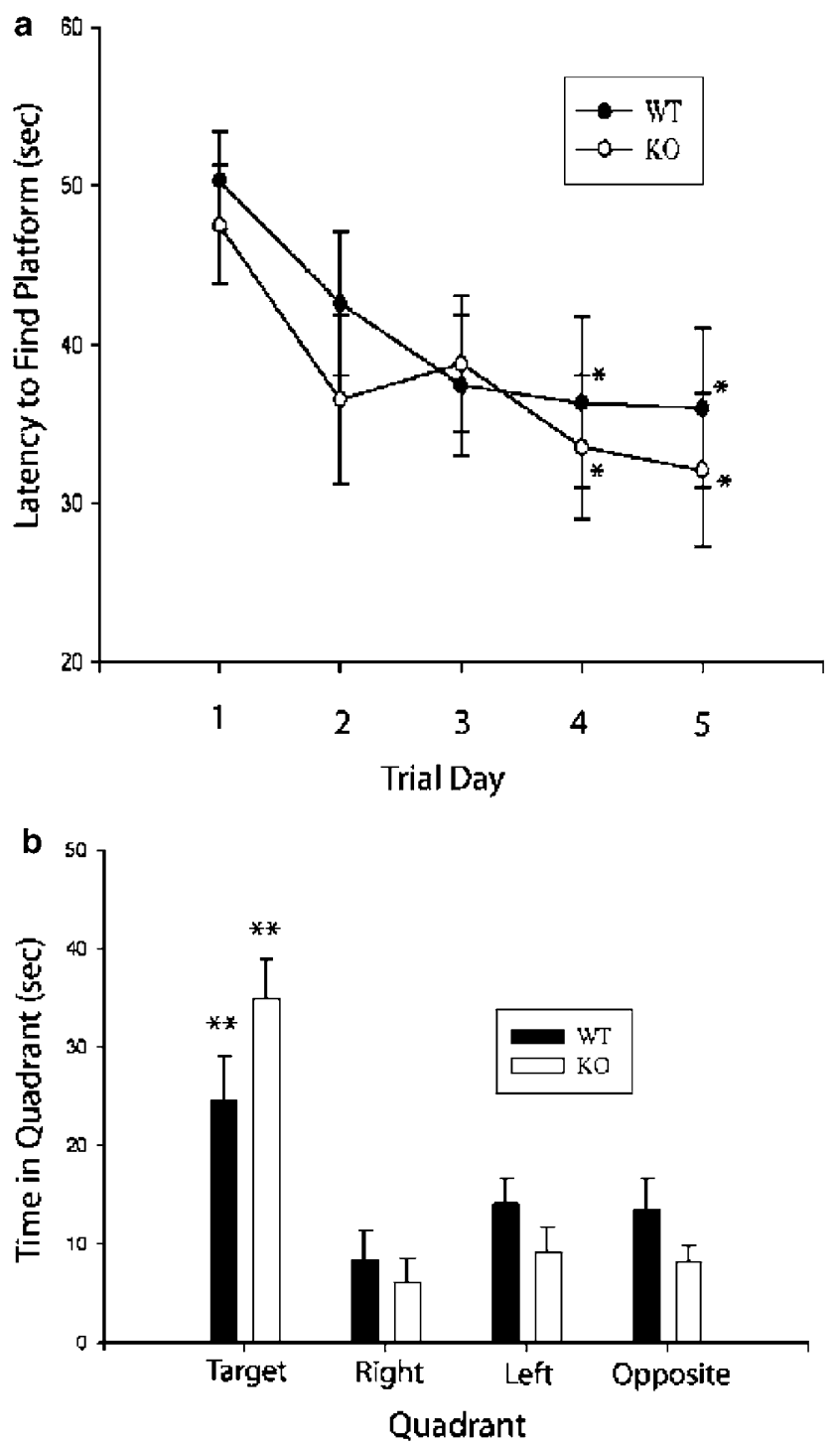

Figure 2 Morris water maze test for learning and memory. There was no difference between the VIaRKO (open circles, $N=9$ ) and the WT (closed circles, $N=8$ ) latency to find the platform on any of the 5 days of training (a). Each day animals were given four trials of $60 \mathrm{~s}$ each to find the submerged platform. The average latency to find the platform for each day is plotted by genotype. Both VIaRKO and WT animals demonstrated a decreased latency to find the platform on trial days 4 and 5 . On day 6 a $60 \mathrm{~s}$ probe trial for retention and retrieval (b) was performed in which the platform was removed and the animals were assessed for time spent swimming in each quadrant. Both VIaRKO (open bars) and WT (filled bars) animals spent significantly more time in the target quadrant as compared to the other quadrants. There was no difference between genotypes. Error bars represent \pm SEM. ${ }^{*} p<0.05$, ** $p<0.001$.

$0.139 \pm 0.011$ (Figure $6 \mathrm{~b}$ ), respectively, and in the V1aRKO subjects was $0.169 \pm 0.04$ and $0.172 \pm 0.013$, respectively (Figure $6 \mathrm{~d})$. The average OTR binding $(\mathrm{dpm} / \mathrm{mg})$ in the lateral septum $(p>0.05)$ and medial amygdala $(p>0.05)$ in WT subjects was $5136.2 \pm 336.2$ and $4565.7 \pm 695.5 \mathrm{dpm} / \mathrm{mg}$, respectively, and in the V1aRKO subjects was $4648.89 \pm 383.6$ and $5534.4 \pm 646.9 \mathrm{dpm} / \mathrm{mg}$, respectively. The lack of V1aRs in the knockouts was confirmed by analyzing V1aR binding in the WT (Figure 6a) and V1aRKO (Figure 6c).
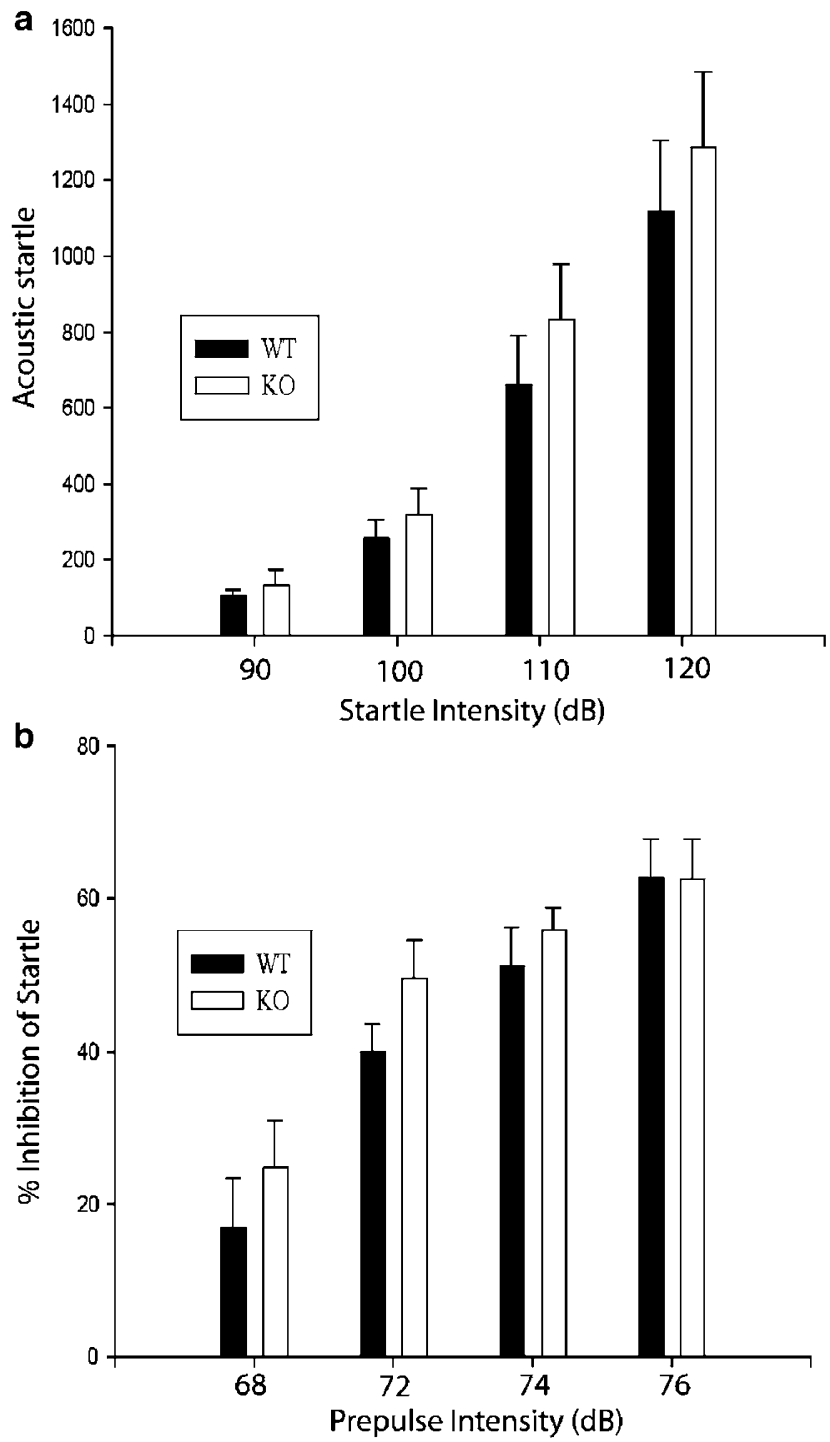

Figure 3 Baseline acoustic startle and PPI. (a) There was no difference between WT (filled bars, $N=8$ ) and VIaRKO (open bars, $N=9$ ) animals' baseline startle at any of the four intensities. (b) There were no genotypic differences in PPI of the startle response at any of the prepulse intensities. Error bars represent + I SEM.

\section{DISCUSSION}

The results of this study definitively establish a role for $\mathrm{V} 1 \mathrm{aR}$ in the regulation of social recognition and anxietyrelated behavior in mice. This is the first report to use mice with a null mutation in the V1aR gene to demonstrate the dependence of social and anxiety-related behavior on this receptor subtype. In addition, these findings support previous findings in which V1aR disruption using antisense oligonucleotides in the lateral septum caused deficits in social recognition and decreased anxiety-related behavior (Landgraf et al, 1995). These findings strongly suggest that VlaR activity is required for the expression of social recognition and plays a significant role in regulating anxiety-like behavior. Previous studies using the V1aRKO have focused solely on immunological differences in $\mathrm{T}$ and B cell development (Hu et al, 2003). 

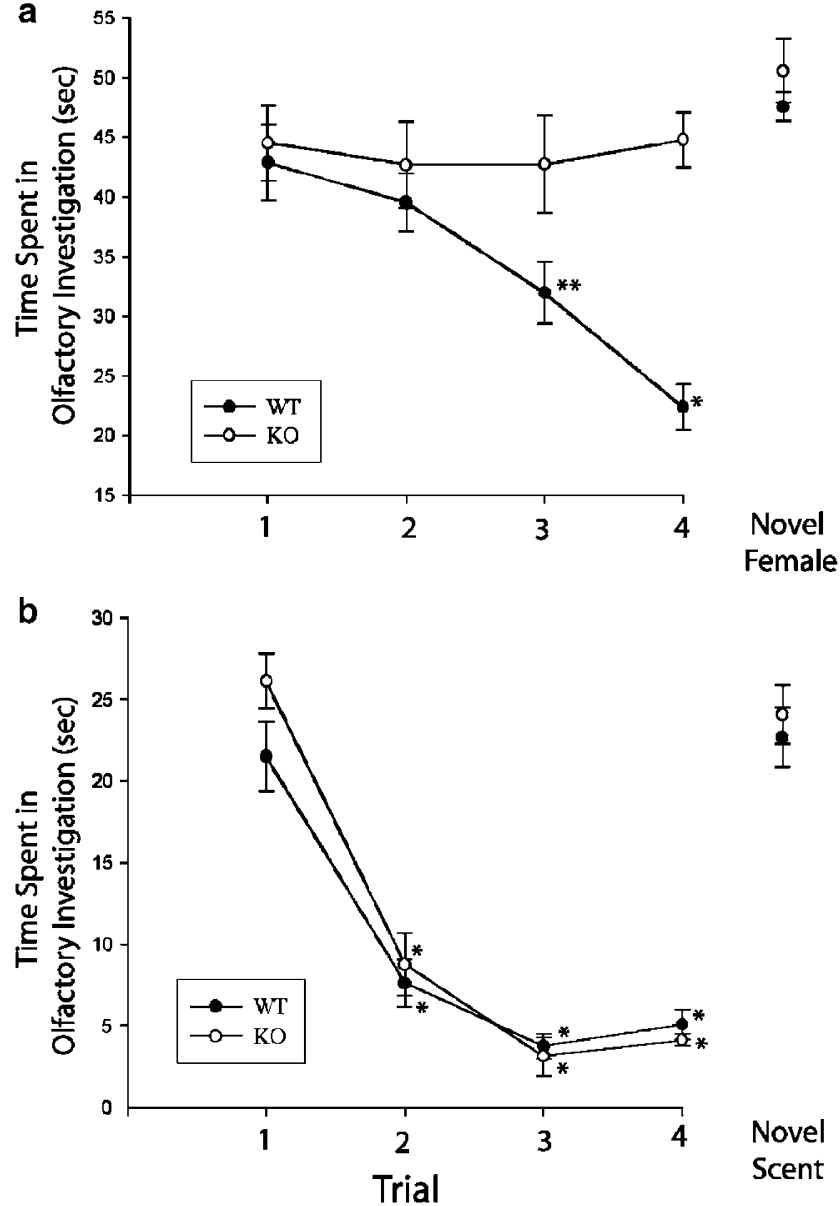

Figure 4 Social recognition and olfactory habituation. (a) Social recognition of $\mathrm{VIaRKO}$ (open circles, $N=9$ ) and $\mathrm{WT}$ (closed circles, $N=8$ ) was measured as a difference in olfactory investigation of the same ovariectomized female during each of four successive I min trials $(I T I=10 \mathrm{~min})$. A fifth dishabituation trial depicts the response to a novel female in a I-min pairing 10 min after the fourth trial. WT mice showed a significant decrease in olfactory investigation after repeated parings of the same female on trials 3 and 4 . VIaRKO mice never showed a decrease in olfactory investigation. (b) Olfactory habituation was measured as a difference in olfactory investigation of an anise scented cotton ball during each of four successive I min trial $(I T|=| 0 \mathrm{~min})$. The fifth, dishabituation trial depicts the response to a coconut scented cotton ball in a I-min exposure $10 \mathrm{~min}$ after the fourth trial. Both $\mathrm{WT}(\mathrm{N}=8)$ and VIaRKO $(N=9)$ mice showed a decrease in investigation upon subsequent presentations of the same scented cotton ball on trials 2,3 , and 4 . ${ }^{*} * 0<0.0$ I, ${ }^{*} p<0.00$ I. Error bars represent \pm SEM. Asterisks represent a significant decrease between each trial as compared to the first trial.

Traditionally research into AVP function has focused on its peripheral actions of fluid homeostasis (via the V2 receptor) and blood pressure control (V1aR) via release from the posterior pituitary (hypothalamic AVP). AVP is also released at the anterior pituitary where it acts on V1bR to help control ACTH release and therefore modulate the hypothalamic-pituitary-adrenal (HPA) axis (Antoni, 1993; de Keyzer et al, 1994). The central and behavioral affects of AVP are likely a result of other AVP systems in the brain. There exists a sexually dimorphic extrahypothalamic AVP circuit that may play a critical role in AVP-mediated behavior (de Vries and Miller, 1998). In this circuit, AVP is
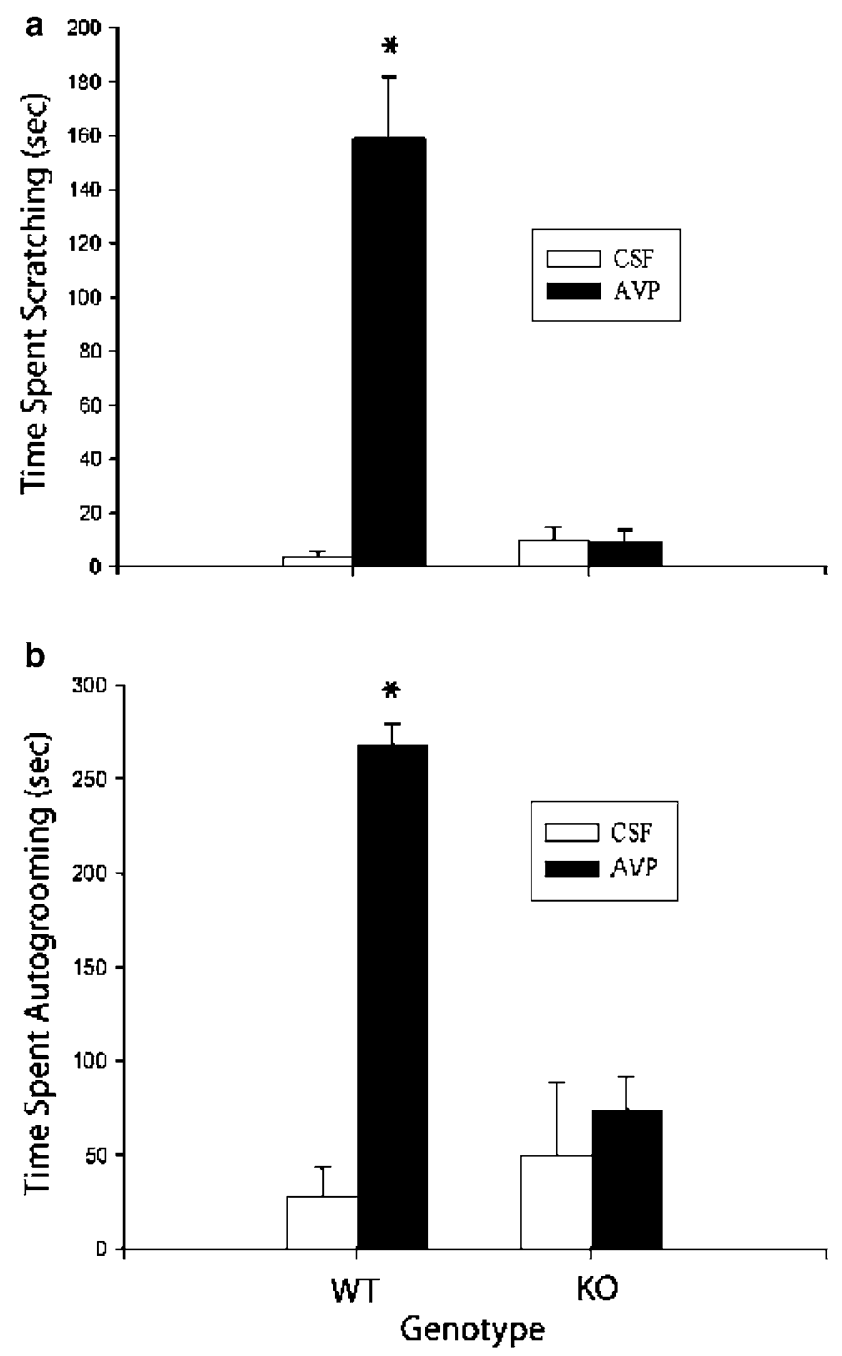

Figure 5 Scratching and autogrooming in response to AVP and CSF i.c.V. injections. WT $(N=8)$ animals spent more time scratching (a) and autogrooming (b) in response to AVP (filled bars) i.c.v. injection compared to vehicle CSF (open bars). There was no effect of AVP treatment in the VI aRKO $(N=9)$ and they did not differ from the WT CSF-treated animals in scratching and autogrooming. Error bars represent + SEM. $* p<0.001$.

synthesized in the medial amygdala and the bed nucleus of the stria terminalis and AVP neurons from these areas project to forebrain structures such as the lateral septum. The lateral septum contains a dense plexus of fibers containing AVP (de Vries and Miller, 1998). Lesions of the lateral septum reduce anxiety-related behavior and septally released AVP has been shown to be causally involved in social recognition and anxiety-related behavior (Menard and Treit, 1996; Landgraf et al, 1998). While there have been recent reports suggesting that V1bRs are located throughout the brain, the V1aR remains the dominant AVP receptor in the lateral septum (Vaccari et al, 1998; Hernando et al, 2001). Such data suggest that localized extrahypothalamic activation of the V1aR may be critical to AVP's effects on social behaviors and anxiety behaviors.

Investigations into the behavioral effects of AVP originally focused on the positive effects of AVP treatment on passive and active avoidance learning and memory 


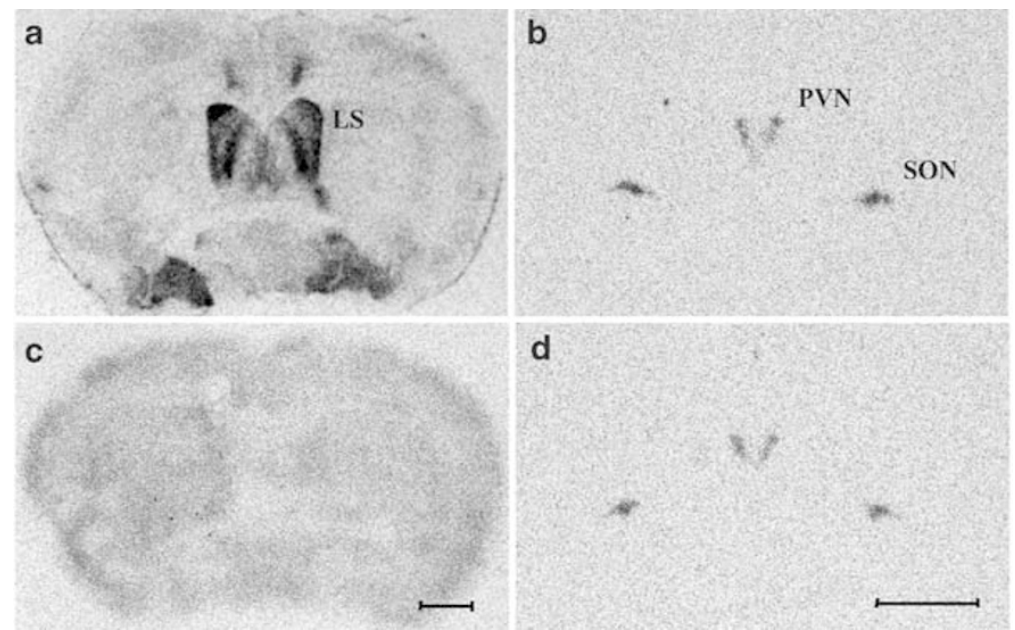

Figure 6 VIaR-binding autoradiograms and AVP in situ illustrating ${ }^{125}$ I linear VI aR antagonist binding in WT (a) and VI aRKO (c) males at the level of the lateral septum and antisense oligo hybridization to AVP mRNA in WT (b) and VI aRKO (d) at the level of the PVN and supraoptic nucleus (SON). Scale bars are equivalent to $1 \mathrm{~mm}$.

(reviewed in van Wimersma Greidanus et al, 1983). However, the evidence for the role of AVP in learning and memory has not been without controversy and many groups have suggested that the behavioral effects of AVP treatment are in fact an indirect behavioral consequence of AVP affecting arousal state and emotionality (Sahgal, 1984; Dantzer, 1998). Due to the aversive nature of passive and active avoidance paradigms, it is difficult to separate out these two possibilities. It has been hypothesized that the relationship between task performance and arousal takes the form of an inverted $U$ shape. A small increase in arousal might result in a facilitation of performance in learning and memory task, while a large increase in arousal might negatively affect task performance (Sahgal, 1984; Dawson et $a l, 1992)$. We attempted to address this controversy by testing the effects of a null mutation in the $V 1 a R$ on emotionality (using anxiety- and depression-like tests), learning and memory that is nonaversive in nature (social recognition), and learning and memory that is slightly aversive but not clearly affected by AVP treatment. Our results suggest that VlaR activation does affect emotionality, but that the learning and memory affects of AVP are more task dependent.

Previous pharmacological investigations, by a variety of laboratories, into the role of the $\mathrm{VlaR}$ in anxiety have yielded a contradictory and inconsistent collection of data. Appenrodt et al (1998), reported a decrease in anxietyrelated behavior after central or peripheral AVP administration but no effect of an AVP antagonist, while Liebsch et al (1996) found no effect of AVP on anxiety behavior, but did find a decrease in anxiety-related behavior after treatment with a V1 receptor antagonist. A third group found that AVP receptor antagonism increased anxiety-like behavior (Liebsch et al, 1996; Appenrodt et al, 1998; Everts and Koolhaas, 1999). The V1bR has also been implicated in anxiety, and treatment with an orally active V1bR antagonist resulted in a decrease in anxiety-related behavior in rats (Griebel et al, 2002). This finding was contradicted by the report that V1bR knockout mice do not show any changes in anxiety-related behavior (Wersinger et al, 2002).
These inconsistencies may be due, in part, to the existence of two AVP receptor subtypes in the brain and the ability of many receptor antagonists to bind either receptor subtype, as well as the related central OTR. The inability to clearly discern the role of the VlaR in anxiety from pharmacological investigations and the confounding results from investigations into the $\mathrm{V} 1 \mathrm{bR}$ prompted the current studies with the V1aRKO mice. The significantly reduced anxietylike behaviors observed in the VlaRKO subjects provide additional, nonpharmacological support for the role of $\mathrm{V} 1 \mathrm{aR}$ in regulating anxiety behavior. Under the conditions used for testing, the WT subjects exhibited elevated levels of anxiety-like behavior relative to other reports (Everts and Koolhaas, 1999). The reasons for this are unclear but could be due to lux levels, time of day, and/or the general conditions in our mouse colony or testing rooms.

While the V1aRKO mice showed a significant decrease in anxiety-like behavior in the elevated plus maze, the open field, and the light/dark box, there was no difference in the forced swim test. The forced swim test is generally considered a measure of depression-like behavior, and not anxiety, due to the ability of antidepressants, but not anxiolytics, to alter swimming behavior (Porsolt et al, 1978). However, previous studies have found septal AVP release in response to the forced swim test and septal infusion of V1 antagonist increases the amount of time in passive (floating) $v s$ active (swimming) behavior (Ebner et al, 1999). We found no genotypic difference in the time spent in passive (floating) $v s$ active (swimming) behavior and thus suggest that the anxiolytic-like effect of the null mutation in the V1aRKO mice is not a result of nonspecific changes in mood. The results of the forced swim task are also further evidence that the null mutation does not affect locomotor activity as both genotypes had similar levels of active (swimming) behavior.

While previous pharmacological studies of AVP have often yielded ambiguous anxiety results, the most consistent data on the effects of AVP is the stimulation of a stereotypy of scratching and autogrooming (Meisenberg, 1988; Moody et al, 1988). I.c.v. infusion of AVP in the WT animals 
resulted in a highly significant increase in both scratching and autogrooming. There was no difference between vehicle treatment in WT subjects and AVP or vehicle treatment in V1aRKO subjects. Some investigators have interpreted scratching and autogrooming as anxiety-related displacement behaviors, however; it would be deficient to not address the possibility that the large increase in scratching and autogrooming seen in the AVP-treated WT animals is a result of an increase in 'itchiness' as apposed to an example of anxiety-related behavior. While this remains a possibility, these affects of AVP have been shown to decrease significantly after treatment with psychotropic agents or in contexts that allow for escape-directed activity and therefore are probably a behaviorally distinct phenomenon (Meisenberg, 1988).

The direct effects of acute AVP treatment on anxiety-like behaviors as assessed by more traditional anxiety tests have been shown to be difficult to detect (Liebsch et al, 1996). The already high levels of anxiety-like behaviors witnessed in the WT and the evidence that prior test experience can alter the expression of anxiety-like behavior after treatment (Holmes et al, 2001), dictated the use of scratching and autogrooming as the primary measure of a behavioral effect of AVP treatment in our studies. The AVP treatment findings support the previous reports of AVP-induced stereotypy of scratching and autogrooming and provide clear demonstration that at least these components of AVPinduced behavior are entirely dependent on V1aR and may have some relevance to the anxiety-related changes observed using the more traditional anxiety tests.

Previous reports on the role of the V1aR in social recognition are more consistent than the anxiety data, with most pharmacological investigations of $\mathrm{V} 1 \mathrm{aR}$ antagonists resulting in impaired social recognition (Everts and Koolhaas, 1997, 1999; Ferguson et al, 2002). Conversely, AVP agonists have a facilitating affect on social memory and can prolong social recognition for as long as $120 \mathrm{~min}$. Normal social recognition lasts between 30 and $60 \mathrm{~min}$ and the facilitating effects of AVP administration appear to improve the consolidation of the social memory given that administration is effective if given after the initial encounter (Dantzer et al, 1987; Le Moal et al, 1987). Increased expression of the V1aR in the lateral septum, using viral vector gene transfer, resulted in a similar facilitation of social recognition (Landgraf et al, 2003). OT has also been shown to be critical to social recognition, and animals lacking OT (OTKO) have severely impaired social memory (Ferguson et al, 2000); however, the effects of OT on social recognition differ from the effects of AVP. OT appears to modulate the acquisition of the social memory and must be present during the initial encounter with the stimulus animal (Ferguson et al, 2001). Further complicating the issue is the finding that V1bRKO animals have mildly impaired social recognition, which presents the possibility that the V1bR is affecting AVP-mediated social recognition (Wersinger et al, 2002). Our current findings that V1aRKO mice have a profound deficit in social recognition, provides definitive evidence that the $\mathrm{V} 1 \mathrm{aR}$ is critical to social recognition. This social deficit is not a result of a general olfactory deficiency, given the ability of these animals to habituate to a nonsocial stimulus, suggesting that olfactory learning in V1aRKO mice is intact and the specific deficit is in the learning and recall of social cues. These results suggest that V1aRs are either involved in the proper processing of olfactory cues or pheromones used to identify individuals, or perhaps in the context specific learning or recall of social olfactory memories.

The extensive circuitry of the AVP systems in the brain presents the possibility that disruption of the V1aRs in our mice might result in other behavioral deficits, which could confound our results. For that reason we conducted several behavioral tests to rule out more global behavioral changes. The VlaRKO mice showed no deficits in spatial learning and memory as measured by the mildly aversive, Morris water maze. Previously only a few studies have found any effects of AVP on spatial memory. Intraseptal and subcutaneous AVP administration retarded acquisition of spatial learning and memory in the Morris water maze and T-maze tasks (McDaniel et al, 1989; Engelmann et al, 1992). In the present study, both genotypes demonstrated normal and statistically similar acquisition, retention, and retrieval of spatial memory.

Other changes in behavioral measures, which could confound our findings were also found to be normal, including olfactory habituation, sensorimotor gating using PPI, and locomotor activity. PPI was included as a control for normal sensory motor gating (SMG). SMG is a neural mechanism that allows the generation of appropriate behavioral responses to incoming sensory cues and the inhibition of extraneous sensory, cognitive, and motor information (Dulawa and Geyer, 2000). Normal sensory motor functioning is critical to many of the behavioral tasks in these experiments. As an important control for PPI, the baseline acoustic startle was also assessed. There were no differences between the WT and V1aRKO at any of the pulse or prepulse frequencies.

Given the role of AVP in suparchiasmatic nucleus (SCN) functioning (de Vries and Miller, 1998) it is possible that some of our behavioral effects could be attributable to changes in circadian rhythm, particularly the anxiety tests, which are based on the animals' natural aversion to bright light. Such an indirect effect cannot be excluded. Furthermore, the constitutive nature of the disruption in the $V 1 a R$ gene could have resulted in the observed abnormal behavioral phenotype, however, previous literature using acute modulation of the V1aR resulted in similar social and anxiety-like changes in behavior (Landgraf et al, 1995). Finally, great care was taken to reduce other possible confounding variables. Anxiety tests were conducted first to prevent any influence from the mildly aversive Morris water maze and PPI. Social recognition and olfactory habituation were conducted at the end of behavior testing but before the pharmacological manipulations. This was due to the 10 days of social isolation required for these tests, and the possible effects this could have on anxiety-like behavior.

The results from the binding and in situ studies suggest that the behavioral effects of the null mutation in the V1aR cannot be attributed to compensatory changes in the expression of AVP or OT or OT receptor binding, as there were no differences in these parameters between genotypes. However other compensatory changes, such as peptide release or increased activation of V1bR or OTR by AVP cannot be completely ruled out, as constitutive mouse 
mutations can result in developmental adaptations and functional compensations.

Our findings provide direct evidence that the $\mathrm{V} 1 \mathrm{aR}$ is critical in olfactory social recognition and anxiety-like behavior. Taken together with the results of the control experiments on learning and memory, sensory processing, and olfaction, these data suggest that AVP is involved in the normal processing of socially relevant cues and responses to anxiety-evoking conditions, but not general cognitive functioning. Our results support previous reports of the importance of the AVP system in these behaviors and may have clinical relevance to the understanding and treatment of similar corresponding disorders in humans. Autism, with its hallmark disturbances in social behavior, has been associated with polymorphisms in the V1aR gene (Kim et al, 2002), suggesting that modulation of this receptor and the AVP system may provide novel therapeutic targets for psychiatric disorders associated with social impairment, such as autism, as well as affective disorders such as anxiety disorder. However, the therapeutic potential of AVP treatment must not be overstated. Social recognition in rodents is a specific olfactory task and is subserved by a clear olfactory circuit important in such species. The effects of AVP in social recognition may not generalize to social engagement in humans, and furthermore, the effects V1aR modulation on anxiety-related behavior might confound the use of AVP focused treatment for social disorders and vice versa. However, the disparate effects of VlaR activation on social- and anxiety-related behavior may be mediated by different neural circuits and AVP focused treatment for one disorder could possibly be separated from effects on other circuits. It is also possible, as discussed previously, that AVP's effects on social recognition and anxiety behaviors are a result of changes in the internal milieu, and that AVP has a more permissive affect on arousal states. However, our findings do suggest that the modulation of the vasopressinergic system may provide a fresh strategy for the treatment of social and affective disorders and further investigation may prove the V1aR and AVP systems to be clinically relevant to these disorders.

\section{ACKNOWLEDGEMENTS}

This research was supported by NIH R01 MH56897 and K02 MH64692 to LJY and NSF STC IBN-9876754 and the Yerkes Center Grant RR00165.

\section{REFERENCES}

Antoni FA (1993). Vasopressinergic control of pituitary adrenocorticotropin secretion comes of age. Front Neuroendocrinol 14: 76-122.

Appenrodt E, Schnabel R, Schwarzberg H (1998). Vasopressin administration modulates anxiety-related behavior in rats. Physiol Behav 64: 543-547.

Bamshad M, Albers HE (1996). Neural circuitry controlling vasopressin-stimulated scent marking in Syrian hamsters (Mesocricetus auratus). J Comp Neurol 369: 252-263.

Dantzer R (1998). Vasopressin, gonadal steroids and social recognition. Prog Brain Res 119: 409-414.

Dantzer R, Bluthe RM, Koob GF, Le Moal M (1987). Modulation of social memory in male rats by neurohypophyseal peptides. Psychopharmacology 91: 363-368.
Dawson GR, Heyes CM, Iversen SD (1992). Pharmacological mechanisms and animal models of cognition. Behav Pharmacol 3: 285-297.

de Keyzer Y, Auzan C, Lenne F, Beldjord C, Thibonnier M, Bertagna $X$ et al (1994). Cloning and characterization of the human V3 pituitary vasopressin receptor. FEBS Lett 356: 215-220.

de Vries GJ, Miller MA (1998). Anatomy and function of extrahypothalamic vasopressin systems in the brain. Prog Brain Res 119: 3-20.

Dulawa SC, Geyer MA (2000). Effects of strain and serotonergic agents on prepulse inhibition and habituation in mice. Neuropharmacology 39: 2170-2179.

Ebner K, Wotjak CT, Holsboer F, Landgraf R, Engelmann M (1999). Vasopressin released within the septal brain area during swim stress modulates the behavioural stress response in rats. Eur J Neurosci 11: 997-1002.

Engelmann M, Bures J, Landgraf R (1992). Vasopressin administration via microdialysis into the septum interferes with the acquisition of spatial memory in rats. Neurosci Lett 142: 69-72.

Engelmann M, Landgraf R (1994). Microdialysis administration of vasopressin into the septum improves social recognition in Brattleboro rats. Physiol Behav 55: 145-149.

Engelmann M, Wotjak CT, Neumann ID, Ludwig M, Landgraf R (1996). Behavioral consequences of intracerebral vasopressin and oxytocin: focus on learning and memory. Neurosci Biobehav Rev 20: 341-358.

Everts HGJ, Koolhaas JM (1997). Lateral septum vasopressin in rats: role in social and object recognition? Brain Res 760: 1-7.

Everts HGJ, Koolhaas JM (1999). Differential modulation of lateral septal vasopressin receptor blockade in spatial-learning, social recognition, and anxiety-related behaviors in rats. Behav Brain Res 99: 7-16.

Ferguson JN, Aldag JM, Insel TR, Young LJ (2001). Oxytocin in the medial amygdala is essential for social recognition in the mouse. J Neurosci 21: 8278-8285.

Ferguson JN, Young LJ, Hearn EF, Matzuk MM, Insel TR, Winslow JT (2000). Social amnesia in mice lacking the oxytocin gene. Nat Genet 25: 284-288.

Ferguson JN, Young LJ, Insel TR (2002). The neuroendocrine basis of social recognition. Front Neuroendocrinol 23: 200-224.

Fernandes C, File SE (1996). The influence of open arm ledges and maze experience in the elevated plus-maze. Pharmacol Biochem Behav 54: 31-40.

Ferris CF, Albers HE, Wesolowski SM, Goldman BD, Luman SE (1984). Vasopressin injected into the hypothalamus triggers a stereotypical behavior in golden hamsters. Science 224: 521-523.

Ferris CF, Meenan DM, Axelson JF, Albers HE (1986). A vasopressin antagonist can reverse dominant/subordinate behavior in hamsters. Physiol Behav 38: 135-138.

Griebel G, Simiand J, Gal CS-L, Wagnon J, Pascal M, Scatton B et al (2002). Anxiolytic- and antidepressant-like effects of the non-peptide vasopressin V1b receptor antagonist, SSR149415, suggest an innovative approach for the treatment of stressrelated disorders. Proc Natl Acad Sci USA 99: 6370-6375.

Hascoët M, Bourin M, Dhonnchadha BÁN (2001). The mouse light-dark paradigm: a review. Prog Neuropsychopharmacol Biol Psychiatry 25: 141-166.

Hernando F, Schoots O, Lolait SJ, Burbach JPH (2001). Immunohistochemical localization of the vasopressin $\mathrm{V} 1 \mathrm{~b}$ receptor in the rat brain and pituitary gland: anatomical support for its involvement in the central effects of vasopressin. Endocrinology 142: $1659-1668$.

Holmes A, Iles JP, Mayell SJ, Rodgers RJ (2001). Prior test experience compromises the anxiolytic efficacy of chlordiazepoxide in the mouse light/dark exploration test. Behav Brain Res 122: $159-167$. 
Hu S-B, Zhao Z-S, Yhap C, Grinberg A, Huang S-P, Westphal H et al (2003). Vasopressin receptor 1a-mediated negative regulation of B cell receptor signaling. J Neuroimmunol 135: 72-81.

Kim S-J, Young LJ, Gonen D, Veenstra-VanderWeele J, Courchesne $\mathrm{R}$, Courchesne $\mathrm{E}$ et al (2002). Transmission disequilibrium testing of arginine vasopressin receptor 1A (AVPR1A) polymorphisms in autism. Mol Psychiatry 7: 503-507.

Landgraf R, Frank E, Aldag JM, Neumann ID, Ren X, Terwilliger $\mathrm{EF}$ et al (2003). Viral vector-mediated gene transfer of the vole V1a vasopressin receptor in the rat septum: improved social discrimination and active social behavior. Eur J Neurosci 18: 403-411.

Landgraf R, Gerstberger R, Montkowski A, Probst JC, Wotjak CT, Holsboer $\mathrm{F}$ et al (1995). V1 vasopressin receptor antisense oligodeoxynucleotide into the septum reduced vasopressin binding, social discrimination abilities, and anxiety-related behavior in rats. J Neurosci 15: 4250-4258.

Landgraf R, Wotjak CT, Neumann ID, Engelmann M (1998). Release of vasopressin within the brain contributes to neuroendocrine and behavioral regulation. Prog Brain Res 119: 201-220.

Le Moal M, Dantzer R, Michaud B, Koob GF (1987). Centrally injected arginine vasopressin (AVP) facilitates social memory in rats. Neurosci Lett 77: 353-359.

Liebsch G, Wotjak CT, Landgraf R, Engelmann M (1996). Septal vasopressin modulates anxiety-related behaviour in rats. $\mathrm{Neu}$ rosci Lett 217: 101-104.

McDaniel WF, Waters PE, Davall EJ (1989). Arginine vasopressin 4-9 retards spatial alternation learning. Neuropeptides 14: 219-224.

Meisenberg G (1988). Vasopressin-induced grooming and scratching behavior in mice. Ann NY Acad Sci 525: 257-269.

Menard J, Treit D (1996). Lateral and medial septal lesions reduce anxiety in the plus-maze and probe-burying tests. Physiol Behav 60: $845-853$.

Moody TW, Merali Z, Crawley JN (1988). The effects of anxiolytics and other agents on rat grooming behavior. Ann NY Acad Sci 252: 281-290.

Morris R (1984). Developments of a water-maze procedure for studying spatial learning in the rat. J Neurosci Methods 11: 47-60.

Nishimori K, Young LJ, Guo Q, Wang Z, Insel TR, Matzuk MM (1996). Oxytocin is required for nursing but is not essential for parturition or reproductive behavior. Proc Natl Acad Sci USA 93: 11699-11704.

Parker KJ, Lee TM (2001). Central vasopressin administration regulates the onset of facultative paternal behavior in microtus pennsylvanicus (meadow voles). Horm Behav 39: 285-294.

Pitkow LJ, Sharer CA, Ren X, Insel TR, Terwilliger EF, Young LJ (2001). Facilitation of affiliation and pair-bond formation by vasopressin receptor gene transfer into the ventral forebrain of a monogamous vole. J Neurosci 21: 7392-7396.

Porsolt RD, Anton G, Blavet N, Jalfre M (1978). Behavioral despair in rats: a new model sensitive to antidepressant treatments. Eur J Pharmacol 47: 379-391.

Sahgal A (1984). A critique of the vasopressin-memory hypothesis. Psychopharmacology 83: 215-228.

Sanchez C, Meier E (1997). Behavioral profiles of SSRIs in animal models of depression, anxiety and aggression. Psychopharmacology 129: 197-205.

Vaccari C, Lalait SJ, Ostrowski NL (1998). Comparative distribution of vasopressin $\mathrm{V} 1 \mathrm{~b}$ and oxytocin receptor messenger ribonucleic acids in brain. Endocrinology 139: 5015-5033.

van Wimersma Greidanus TB, Maigret C (1996). The role of limbic vasopressin and oxytocin in social recognition. Brain Res 713: 153-159.

van Wimersma Greidanus TB, van Ree JM, de Wied D (1983). Vasopressin and memory. Pharmacol Ther 20: 437-458.

Wersinger SR, Ginns EI, O'Carroll AM, Lolait SJ, Young III WS (2002). Vasopressin V1b receptor knockout reduces aggressive behavior in male mice. Mol Psychiatry 7: 975-984.

Winslow JT, Hastings N, Carter CS, Harbaugh CR, Insel TR (1993). A role for central vasopressin in pair bonding in monogamous prairie voles. Nature 365: 545-548.

Young LJ, Huot B, Nilsen R, Wang Z, Insel TR (1996). Species differences in central oxytocin receptor gene expression: comparative analysis of promotor sequences. J Neuroendocrinol 8: $777-783$.

Young LJ, Nilsen R, Waymire KG, MacGregor GR, Insel TR (1999). Increased affiliative response to vasopressin in mice expressing the V1a receptor from a monogamous vole. Nature 400: 766-768.

Young LJ, Winslow JT, Nilsen R, Insel TR (1997). Species differences in V1a receptor gene expression in monogamous and non-monogamous voles: behavioral consequences. Behav Neurosci 111: 599-605. 